\title{
Evaluation of bleeding risk and measurement methods in dental patients
}

\author{
Aranza Cañigral ${ }^{1}$, Francisco-Javier Silvestre ${ }^{2}$, Guillermo Cañigral ${ }^{3}$, Manuel Alós ${ }^{4}$, Ariadna Garcia-He- \\ rraiz ${ }^{1}$, Andres Plaza ${ }^{5}$ \\ ${ }^{1}$ Dental surgeon. Stomatology Unit. Dr. Peset University Hospital (Valencia) \\ ${ }^{2}$ Assistant Professor. Department of Stomatology. University of Valencia. Head of the Stomatology Unit. Dr. Peset University \\ Hospital (Valencia) \\ ${ }^{3}$ Head of the Service of Hematology. Castellón General Hospital (Castellón) \\ ${ }^{4}$ Head of the Service of Pharmacy. Castellón General Hospital (Castellón) \\ ${ }^{5}$ Associate Professor. Department of Stomatology. University of Valencia (Valencia). Spain
}

Correspondence:

Departament d'Estomatologia

Clinica Odontologica

Universitat de València

C/ Gascó Oliag 1

46010 - Valencia (Spain)

francisco.silvestre@uv.es

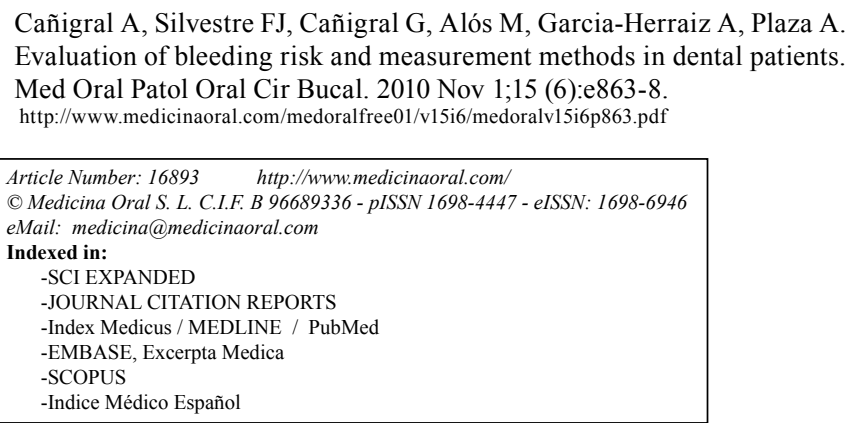

\begin{abstract}
Objective: The present study explores bleeding manifestations in routine dental surgical procedures, evaluates the influence of antithrombotic drugs upon bleeding risk, and validates the efficiency of a clinical method for the measurement of bleeding.

Material and method: A prospective observational study was made involving a cohort of 99 patients in the setting of normal clinical practice, with the added conduction of prior hematological tests including baseline hemostasis and platelet function, based on a new method (Multiplate System $®$ ). For evaluation of the bleeding manifestations, a clinical method was selected that evaluates bleeding on the basis of its duration and the hemostatic measures needed to resolve the problem.

Results: Almost one-third of the patients (27.3\%) were receiving treatment with oral antiplatelet drugs, while $19.2 \%$ received oral anticoagulants and $9 \%$ received combined therapy with acetylsalicylic acid plus clopidogrel. In turn, an $8 \%$ incidence of moderate bleeding episodes was detected correlated to the ASPI platelet function test and to advanced patient age.

Conclusion: The incorporation of platelet function tests increases the safety of oral surgery in elderly patients subjected to antiplatelet treatment, particularly with acetylsalicylic acid and clopidogrel.
\end{abstract}

Key words: Oral surgery, bleeding risk, antithrombotic treatment, antiplatelet drugs. 


\section{Introduction}

Bleeding is one of the most frequent complications in dental practice, and can be produced by the use of invasive techniques or the existence of alterations in hemostasis of a primary (congenital) or secondary (acquired) nature associated with certain systemic illnesses and/or the administration of certain drugs.

Many dental patients are elderly, with a high prevalence of chronic systemic diseases and a frequent need for multiple drug treatments. In this context, attention is drawn to the administration of antithrombotic drugs, which is growing at an annual rate of over $10 \%$. This in turn leads to an increase in the detection of secondary hemostatic alterations that complicate dental treatment and the management of these patients, with an increase in the risk of bleeding (1).

The blood is a fluid tissue, and the hemostatic system is in charge of preventing its extravasation in the event of blood vessel damage. Classically, hemostasis is characterized by two phases: primary and secondary. The primary phase fundamentally involves the vascular- and platelet-mediated processes that ensure the early arrest of bleeding as a result of early clot formation. Secondary hemostasis in turn generates a fibrin clot to secure definitive sealing of the damaged blood vessel and repair. In fact, under normal conditions all these processes coexist in a permanent balance between coagulation / anticoagulation and fibrinolysis / antifibrinolysis, and bleeding or thrombosis result when this balanced is inclined in favor of one process or the other.

A well compiled clinical history should detect any bleeding antecedents, allowing the dental surgeon to obtain an idea of the type of hemostatic alterations the patient may have, and to determine whether such alterations are of a point nature attributable to purely local factors, or whether the patient has a constitutional tendency towards bleeding.

Different criteria are used in general surgical procedures to assess the intensity of perioperative bleeding, though its estimation is not very rigorous in most studies, and consensus has not been established on this issue in the scientific community. One of the most widely used indicators is the Bleeding Severity Index (BSI), which assesses the need for blood product transfusions and stratifies the severity of bleeding into three levels according to the blood product units transfused: mild (transfusion of 1 to $<2$ red cell concentrate units), moderate (transfusion of 2 to $<3$ units) or severe (transfusion of 3 or more units)(2).

In relation to oral procedures, most of the reviewed studies make no mention of the methodology used to measure bleeding. Nevertheless, those methods that have been described in the literature involve different approaches. Vicente Barrero et al. (3) divide postoperative bleeding into three levels: mild when bleeding last less than 5 minutes, moderate when it last more than 5 minutes, and intense when transfusion proves necessary. On the other hand, Morimoto et al. (4) make a distinction between postoperative bleeding that subsides upon applying pressure with a piece of gauze, and severe bleeding that does not subside with pressure and which requires intervention by the oral surgeon to ensure hemostasis (compression, removal of the hematoma, curettage, the application of oxidized cellulose, suturing, etc.). Krishnan et al. (5) define prolonged postoperative bleeding as bleeding that persists despite the application of gauze and pressure in the extraction socket during 30 minutes. In turn, clinically significant bleeding is taken to be bleeding that persists 12 hours after the procedure, gives rise to patient consultation or a visit to the emergency room, produces important hematoma in the soft tissues, or requires blood transfusion. On the other hand, Al-Belasy et al. (6) used a more precise method in which postoperative bleeding is divided into three levels: mild bleeding that subsides in under 20 minutes upon applying pressure with a piece of sterile gauze; moderate bleeding that does not subside with such measures and requires tranexamic acid irrigation for two minutes and tamponade with tranexamic acid impregnated gauze for 20 minutes; and severe bleeding that requires further suturing, the administration of vitamin K, or the infusion of fresh frozen plasma.

The frequency and diagnostic importance of bleeding manifestations of the oral cavity that exceed the setting of dental practice makes it necessary to use and evaluate objective, precise and effective bleeding measurement methods, with an analysis of the repercussion of antithrombotic drugs upon bleeding risk.

The aims of the present study are to explore bleeding manifestations in dental surgical procedures, evaluate the influence of antithrombotic drugs upon bleeding risk, and validate the efficiency of a clinical method for the measurement of bleeding after dental extraction or oral surgery.

\section{Materials and Methods}

A prospective observational study was made involving a cohort of 99 patients subjected to successive randomized recruitment without limitations in terms of severity or complexity. The patients were enrolled from among the subjects seen in the Stomatology Units of Dr. Peset University Hospital (Valencia, Spain) and Castellón General Hospital (Castellón, Spain). Only terminally ill patients were excluded, and the study was approved by the ethics committees of the two hospital centers.

All patients were attended according to the routine clinical practice protocols applied in the respective hospital units. Those patients receiving antiplatelet medication and programmed for simple tooth extraction were subjected to dental surgery without suppression of the 
antiplatelet medication. In the case of complex procedures (multiple extractions, impacted dental surgery, biopsies) or combined antiplatelet treatment, the patients were referred to the Service of Hematology to plan the management approach. In the case of patients receiving oral anticoagulation and programmed for simple dental procedures, the international normalized ratio (INR) was monitored, and if the anticoagulation values proved compatible with surgery, the dental operation was carried out. In the case of complex procedures, the patients were likewise referred to the Service of Hematology for programming of the required anticoagulation regimen. On the first visit, a clinical history was compiled, and blood tests were requested, comprising a complete blood count, Quick index, INR, activated partial thromboplastin time (aPTT), fibrinogen and platelet function tests (TRAP test, ASPI test, ADP test, ADPHS test).

The following methodology was used: an ADVIA 120 automatic analyzer ${ }^{\circledR}$ was used for the complete blood count; the coagulation parameters were established with an ACL automatic coagulometer ${ }^{\circledR}$ (TOP $3 \mathrm{G}$ version 28.2); and the platelet function tests were performed with the Multiplate System analyzer ${ }^{\circledR}$, with four different and simultaneous tests for each patient sample: TRAP test, ASPI test, ADP test and ADPHS test. The TRAP test allows the detection of glycoprotein IIb/IIIa antagonists (abciximab, tirofiban and eptifibatide) even in samples exposed to acetylsalicylic acid (ASA, aspirin) and clopidogrel. The ASPI test is a specific assay for the discrimination of acetylsalicylic acid, while the ADP test detects the presence of clopidogrel, prasugrel and ticlopidine. Finally, the ADPHS test comprises the addition of an endogenous inhibitor to the ADP test, making it more sensitive in detecting clopidogrel and related drugs. This technology is simple, rapid and convenient for application as part of the preoperative patient workup. The system is compact, and its 5 channels applying different stimulants allow the performance of parallel tests in a sequence lasting 10-15 minutes. Processing requires $0.3 \mathrm{ml}$ of whole blood per test, and is carried out by an integrated computer system with automatic pipetting.

Evaluation of the bleeding manifestations was based on an easily quantifiable and objective clinical method in which bleeding is assessed according to its duration and the hemostatic measures needed to stop bleeding. Accordingly, three levels of bleeding intensity were defined: mild (subsides in under 10 minutes with sterile gauze tamponade), moderate (subsides with tamponade plus local hemostatic treatment in under 60 minutes) and severe (bleeding for over 60 minutes and requiring other medical or surgical management).

For data collection, a form was developed documenting different demographic parameters, patient antecedents, acquired and congenital bleeding risk factors, simple or complex dental procedures, bleeding manifestations (mild, moderate, severe), complications, and a range of laboratory test data.

The statistical analysis was carried out using the SPSS ${ }^{\circledR}$ version 15.0 statistical package for Microsoft Windows. The descriptive study of the continuous variables was based on the measures of central tendency and dispersion (mean and standard deviation), while the categorical variables were reported as absolute and percentage frequencies.

The evaluation of bleeding risk was based on logistic regression analysis involving the continuous and categorical variables. The results of the logistic regression analysis were presented as the Odds Ratio (OR) and corresponding $95 \%$ confidence interval $(95 \% \mathrm{CI})$. Statistical significance was accepted for $\mathrm{p}<0.05$ in all tests.

\section{Results}

The mean age of the patients $(\mathrm{n}=99)$ was $57.7 \pm 19.9$ years (range 15-93). There was a slight female predominance (51.5\% versus $48.5 \%$ males). The biometric profile of the study sample revealed the presence of two large groups corresponding to the 30-40 years age interval and particularly to the 60-80 years age interval.

In relation to the disease antecedents, which moreover were those expected of elderly patients, the most common problems were cardiovascular diseases (40.4\%), followed by arterial hypertension (38.4\%). In turn, $11.1 \%$ of the patients were diabetic, $10 \%$ suffered renal failure, $2 \%$ had neoplastic processes, and $5.1 \%$ presented thromboembolic complications (Table 1).

As a result of the mentioned comorbidities, the patients showed an important use of drug substances. The mean number of drugs per patient was 3.24 (range $0-11)$. Twenty-seven percent of the patients used oral antiplatelet drugs. Specifically, 9\% received combined therapy with ASA plus clopidogrel, $17.2 \%$ used ASA, $10.1 \%$ received clopidogrel, $20 \%$ used low molecular weight heparin (LMWH), $19.27 \%$ were administered oral anticoagulants, and $15.2 \%$ received nonsteroidal antiinflammatory drugs (NSAIDs) other than ASA (Table 1). These percentages were significantly higher than those reported for the general population, and are explained by the high prevalence of comorbid conditions and the advanced age of the subjects.

A total of 99 dental procedures were carried out, of which 51 were simple procedures and 38 complex procedures (impacted tooth surgery and multiple extractions). Most of the bleeding manifestations (92\%) were classified as mild. There were 8 cases of moderate bleeding that subsided with the adoption of local hemostatic measures in under 60 minutes (Table 2). Of the 8 patients who suffered moderate bleeding, 5 were receiving treatment with oral antiplatelet drugs, including four with combined ASA and clopidogrel therapy, while one 
received ASA in monotherapy. In turn, three patients were treated with LMWH, two with other NSAIDs, and only one with oral anticoagulants. On the other hand, one patient presented a history of diabetes mellitus, four had arterial hypertension, and 5 suffered cardiovascular alterations.

The blood count and coagulation parameters showed no significant correlation to bleeding risk.
In relation to moderate bleeding risk following the dental procedure and patient age, the logistic regression analysis showed age to influence risk, with OR 1.05 (95\%CI: 0.99-1.11). Although statistical significance was not reached, a clear tendency was noted. In turn, the logistic regression analysis showed the variable ASPI to be significantly correlated to bleeding, with OR 0.97 (95\%CI: 0.94-0.99, $\mathrm{p}=0.038)$.

Table 1. Disease antecedents, antithrombotic treatment and NSAIDs.

\begin{tabular}{|l|l|}
\hline Disease antecedents & Frequency \\
\hline Diabetes mellitus & $11.1 \%$ \\
\hline Arterial hypertension & $38.4 \%$ \\
\hline Renal failure & $10.1 \%$ \\
\hline Neoplasm & $2 \%$ \\
\hline Cardiovascular alterations & $40.4 \%$ \\
\hline Thromboembolic complications & $5.1 \%$ \\
\hline $\begin{array}{l}\text { Antithrombotic treatment and } \\
\text { NSAIDs }\end{array}$ & Frequency \\
\hline ASA & $17.2 \%$ \\
\hline Clopidogrel & $10.1 \%$ \\
\hline ASA + clopidogrel & $9.1 \%$ \\
\hline Oral anticoagulants & $19.2 \%$ \\
\hline LMWH & $20.2 \%$ \\
\hline NSAIDs & $15.2 \%$ \\
\hline
\end{tabular}

ASA $=$ acetylsalicylic acid

LMWH = low molecular weight heparin

NSAIDs $=$ nonsteroidal antiinflammatory drugs

Table 2. Relationship between bleeding and antithrombotic treatment and NSAIDs.

\begin{tabular}{|l|c|c|c|c|c|c|}
\hline Bleeding & No. patients & ASA & Clopidogrel & LMWH & Oral anticoagulants & NSAIDs \\
\hline Mild & 91 & 12 & 6 & 17 & 18 & 13 \\
\hline Moderate & 8 & 5 & 4 & 3 & 1 & 2 \\
\hline Severe & 0 & ------ & ------ & ------ & ------ & ------ \\
\hline
\end{tabular}

ASA $=$ acetylsalicylic acid

$\mathrm{LMWH}=$ low molecular weight heparin

NSAIDs $=$ nonsteroidal antiinflammatory drugs 


\section{Discussion}

A relevant finding in this study has been the detection of an $8 \%$ incidence of moderate bleeding episodes, significantly related to the ASPI platelet function test, and the tendency of older patients to show an increased risk of moderate bleeding. This is particularly important considering that one-third of the patients were receiving treatment with oral antiplatelet drugs, and that $9 \%$ of these subjects were using combined treatment with ASA and clopidogrel. In coincidence with the general population, most of our patients tended to show blood clotting in under 10 minutes after tooth extraction - such bleeding being classified as mild. Perhaps as a consequence of the patient sample size involved, no cases of severe bleeding were recorded - such problems almost always being associated with alterations of hemostasis and/or local pathological alterations of the treated tissues (hemangioma, important local inflammation).

Of the total dental procedures, more than a third (38\%) were complex (impacted tooth surgery, multiple extractions). In this setting, of the 8 moderate bleeding episodes, three were recorded in patients subjected to complex procedures and 5 in simple extractions - no significant correlation being established between the complexity of the procedure and moderate bleeding risk. This incidence of moderate bleeding was related to the rigorous method used to detect and register such episodes. The number of patients with moderate bleeding goes not allow us to draw conclusions, though particularly older age and the use of ASA and/or NSAIDs up until the time of the dental operation appear to be bleeding risk factors in patients subjected to oral surgery especially among subjects over 65 years old (7). In relation to bleeding and the results obtained in patients subjected to antiplatelet treatment, it should be taken into account that the evaluation of platelet function was made one week before surgery, and that with the exception of ASA - which was not suspended in the case of simple dental procedures - treatment with clopidogrel and combined treatment with ASA and clopidogrel was always either modified or suppressed. This systematic approach was in line with the protocols normally used in both study centers.

As regards the analysis of platelet function, a new technique was used: the Multiplate System ${ }^{\circledR}$ analyzer, based on platelet impedance aggregometry. For years, the first and only option for evaluating platelet function was bleeding time. The impossibility of correct standardization, and particularly the patient discomfort caused (skin and blood vessel damage), gradually lessened the use of this technique. Posteriorly, the need to evaluate platelet function led to the development of technologies such as the PFA-100, a precise, convenient and reliable method that assesses platelet function globally and automatically in citrated whole blood $(8,9)$. However, this system does not detect the effect of certain antiplatelet drugs upon platelet function (10-12).

A recent introduction on the market has been the mentioned Multiplate System ${ }^{\circledR}$ analyzer, which uses different tests to generate reliable information on platelet function and on the effects of antiplatelet treatment. Recent studies support the usefulness of this new technology in discriminating among platelet functional alterations - particularly those attributable to the use of antiplatelet drugs (13-15).

At present, the usual approach in the perioperative management of antiplatelet treatment is to carry out simple extraction procedures without suppressing the antiplatelet medication (16-19). In the case of complex procedures or of combined antiplatelet treatment, the patient should be referred to the Service of Hematology for evaluation of the best approach (20). This sometimes implies delays in oral surgery that may not be justified in all cases. Likewise, no platelet function tests are normally carried out in elderly patients, despite the fact that the present study suggests that advanced age influences at least one of the investigated platelet function tests, with an increase in the risk of bleeding.

A significant finding of this study is the fact that $8 \%$ of the patients subjected to oral surgery suffered moderate bleeding. The risk of bleeding was significantly correlated to the ASPI test, and advanced age showed a nearsignificant association to moderate bleeding risk. As a result, and in contrast to the tendency seen for patient age, an increased ASPI test value indicates increased patient protection against bleeding (20).

In conclusion, the results of this study suggest that incorporation of the new platelet function tests to the preoperative workup of certain complex dental patients seen in the hospital setting may increase the efficiency and safety of the surgical procedures.

Likewise, the study has validated the usefulness of the proposed new bleeding measurement method in oral surgery.

\section{References}

1. Van Diermen DE, Aartman IH, Baart JA, Hoogstraten J, Van der Waal I. Dental management of patients using antithrombotic drugs: critical appraisal of existing guidelines. Oral Surg Oral Med Oral Pathol Oral Radiol Endod. 2009;107:616-24.

2. Landefeld CS, Anderson PA, Goodnough LT, Moir TW, Hom DL, Rosenblatt MW, et al. The bleeding severity index: validation and comparison to other methods for classifying bleeding complications of medical therapy. J Clin Epidemiol. 1989;42:711-8.

3. Vicente Barrero M, Knezevic M, Tapia Martín M, Viejo Llorente A, Orengo Valverde JC, García Jiménez F, et al. Oral surgery in patients undergoing oral anticoagulant therapy. Med Oral. 2002;7:63-6, 67-70.

4. Morimoto Y, Niwa H, Minematsu K. Hemostatic management of tooth extractions in patients on oral antithrombotic therapy. J Oral Maxillofac Surg. 2008;66:51-7.

5. Krishnan B, Shenoy NA, Alexander M. Exodontia and antiplatelet therapy. J Oral Maxillofac Surg. 2008;66:2063-6. 
6. Al-Belasy FA, Amer MZ. Hemostatic effect of n-butyl-2-cyanoacrylate (histoacryl) glue in warfarin-treated patients undergoing oral surgery. J Oral Maxillofac Surg. 2003;61:1405-9.

7. Nematullah A, Alabousi A, Blanas N, Douketis JD, Sutherland SE. Dental surgery for patients on anticoagulant therapy with warfarin: a systematic review and meta-analysis. J Can Dent Assoc. 2009;75:41. 8. Arrieta Blanco JJ, Bartolomé Villar B, Juzgado A, Mourelle Martínez R. Assessment of PFA-100 system for the measurement of bleeding time in oral surgery. Med Oral Patol Oral Cir Bucal. 2006;11:E514-9.

9. Goldenberg NA, Jacobson L, Manco-Johnson MJ. Brief communication: duration of platelet dysfunction after a 7-day course of Ibuprofen. Ann Intern Med. 2005;142:506-9.

10. Dyszkiewicz-Korpanty A, Olteanu H, Frenkel EP, Sarode R. Clopidogrel anti-platelet effect: an evaluation by optical aggregometry, impedance aggregometry, and the platelet function analyzer (PFA100). Platelets. 2007;18:491-6.

11. Madsen EH, Schmidt EB, Maurer-Spurej E, Kristensen SR. Effects of aspirin and clopidogrel in healthy men measured by platelet aggregation and PFA-100. Platelets. 2008;19:335-41.

12. Mueller T, Dieplinger B, Poelz W, Haltmayer M. Utility of the PFA-100 instrument and the novel multiplate analyzer for the assessment of aspirin and clopidogrel effects on platelet function in patients with cardiovascular disease. Clin Appl Thromb Hemost. 2009;15:652-9.

13. Mengistu AM, Wolf MW, Boldt J, Röhm KD, Lang J, Piper SN. Evaluation of a new platelet function analyzer in cardiac surgery: a comparison of modified thromboelastography and whole-blood aggregometry. J Cardiothorac Vasc Anesth. 2008;22:40-6.

14. Siller-Matula JM, Spiel AO, Lang IM, Kreiner G, Christ G, Jilma B. Effects of pantoprazole and esomeprazole on platelet inhibition by clopidogrel. Am Heart J. 2009;157:148.e1-5.

15. Sibbing D, Schulz S, Braun S, Morath T, Stegherr J, Mehilli J, et al. Antiplatelet effects of clopidogrel and bleeding in patients undergoing coronary stent placement. J Thromb Haemost. 2010;8:250-6.

16. Brennan MT, Valerin MA, Noll JL, Napeñas JJ, Kent ML, Fox PC, et al. Aspirin use and post-operative bleeding from dental extractions. J Dent Res. 2008;87:740-4.

17. Partridge CG, Campbell JH, Alvarado F. The effect of platelet-altering medications on bleeding from minor oral surgery procedures. J Oral Maxillofac Surg. 2008;66:93-7.

18. Madan GA, Madan SG, Madan G, Madan AD. Minor oral surgery without stopping daily low-dose aspirin therapy: a study of 51 patients. J Oral Maxillofac Surg. 2005;63:1262-5.

19. Grines CL, Bonow RO, Casey DE Jr, Gardner TJ, Lockhart PB, Moliterno DJ, et al. Prevention of premature discontinuation of dual antiplatelet therapy in patients with coronary artery stents: a science advisory from the American Heart Association, American College of Cardiology, Society for Cardiovascular Angiography and Interventions, American College of Surgeons, and American Dental Association, with representation from the American College of Physicians. J Am Dent Assoc. 2007;138:652-5.

20. Shah PR, Yepes JF, Valenza JA. Combination of aspirin and clopidogrel for the prevention of thrombosis: implications for the dental practitioner. Gen Dent. 2007;55:517-22. 